\title{
Solution and Stability of the Multiquadratic Functional Equation
}

\author{
Xiaopeng Zhao, ${ }^{1,2}$ Xiuzhong Yang, ${ }^{3}$ and Chin-Tzong Pang ${ }^{4}$ \\ ${ }^{1}$ Department of Mathematics, Zhejiang University, Hangzhou 310027, China \\ ${ }^{2}$ Department of Applied Mathematics, National Sun Yat-sen University, Kaohsiung 80424, Taiwan \\ ${ }^{3}$ College of Mathematics and Information Science, Hebei Normal University and Hebei Key Laboratory of \\ Computational Mathematics and Applications, Shijiazhuang 050024, China \\ ${ }^{4}$ Department of Information Management, Yuan Ze University, Chungli 32003, Taiwan
}

Correspondence should be addressed to Chin-Tzong Pang; imctpang@saturn.yzu.edu.tw

Received 25 August 2013; Accepted 10 September 2013

Academic Editor: Chi-Ming Chen

Copyright (C) 2013 Xiaopeng Zhao et al. This is an open access article distributed under the Creative Commons Attribution License, which permits unrestricted use, distribution, and reproduction in any medium, provided the original work is properly cited.

We consider the multiquadratic functional equation. We establish its general solution and provide a characterization for this functional equation. Finally, we prove the Hyers-Ulam-Rassias stability of this functional equation.

\section{Introduction}

In 1940, Ulam [1] gave a talk before the Mathematics Club of the University of Wisconsin, in which he discussed a number of unsolved problems. The stability of a functional equation originated from a question raised by Ulam: "when is it true that the solution of an equation differing slightly from a given one must of necessity be close to the solution of the given equation?" This question was solved by Hyers [2] in the case of the approximately additive functions between Banach spaces. In 1978, Rassias [3] provided a generalized version of Hyers' result by allowing the Cauchy difference to be unbounded. The paper of Rassias [3] has provided a lot of influence in the development of the stability of functional equations, and this new concept is known as generalized Hyers-Ulam-Rassias stability or Hyers-Ulam-Rassias stability. Since then, the stability problems have been widely studied and extensively developed by many authors for a number of functional equations; see, for example, [4-10] and the books [11-14].

The functional equation

$$
f(x+y)+f(x-y)=2 f(x)+2 f(y)
$$

is called the quadratic functional equation, and every solution of the quadratic functional equation is said to be a quadratic function. It is well known that a quadratic function $f: E_{1} \rightarrow$ $E_{2}$ between vector spaces can be expressed by a symmetric biadditive (i.e., additive for each fixed one variable) function $B: E_{1} \times E_{1} \rightarrow E_{2}$. On the other hand, the stability problem for the quadratic functional equation has been studied by many mathematicians under various degrees of generality imposed on the equation or on the underlying space; see, for example, [15-20] and the references therein.

In [21], Park and Bae obtained the general solution and the generalized Hyers-Ulam-Rassias stability of the biquadratic functional equation. Let $X$ and $Y$ be vector spaces. Recall from [21] that a mapping $f: X \times X \rightarrow Y$ is called biquadratic if $f$ satisfies the system of equations

$$
\begin{aligned}
& f(x+y, z)+f(x-y, z)=2 f(x, z)+2 f(y, z) \\
& f(x, y+z)+f(x, y-z)=2 f(x, y)+2 f(x, z)
\end{aligned}
$$

for all $x, y, z \in X$; that is, $f$ is quadratic for each fixed one variable.

A general version of the biquadratic functional equation is the multiquadratic functional equation. Recall from [22] that a mapping $f: V^{n} \rightarrow W$, where $V$ is a commutative group, $W$ is a linear space, and $n \geq 2$ is an integer, is called multiquadratic if it is quadratic in each variable. On the other hand, for more details about the multiadditive (resp., the 
multi-Jensen mappings) (i.e., mappings satisfying Cauchy's (resp., Jensen's) functional equation in each variable) and the stability for them, one can see [23-28] and the references given there.

The stability of the multiquadratic functional equation was also studied by some authors. For example, Park [29] proved the stability of the multiquadratic functional equation in Banach spaces. Ciepliński [22] proved the stability of this functional equation in complete non-Archimedean spaces as well as in Banach spaces but using the fixed point method. However, to our knowledge, not many results are known about the solution of this functional equation.

In the present paper, we establish the general solution of the multiquadratic functional equation and provide a sufficient and necessary condition for a mapping to be multiquadratic. Finally, we prove its Hyers-Ulam-Rassias stability.

\section{General Solution}

Throughout this section, let $V$ and $W$ be vector spaces, and let $n$ be a positive integer. We begin with the following useful proposition.

Proposition 1 (see [11]). A function $f: V \rightarrow W$ is quadratic if and only if there exists a unique symmetric biadditive function $B: V \times V \rightarrow W$ such that $f(x)=B(x, x)$ for any $x \in$ $V$. The biadditive function $B$ is given by

$$
B(x, y)=\frac{1}{4}[f(x+y)-f(x-y)] \quad \forall x, y \in V .
$$

In the following, we give the general solution of the multiquadratic functional equation.

Theorem 2. A mapping $f: V^{n} \rightarrow W$ is multiquadratic if and only if there exists a multiadditive mapping $M: V^{2 n} \rightarrow W$ such that

$$
f\left(x_{1}, x_{2}, \ldots, x_{n}\right)=M\left(x_{1}, x_{1}, x_{2}, x_{2}, \ldots, x_{n}, x_{n}\right)
$$

for all $x_{1}, \ldots, x_{n} \in V$, and $M$ satisfies the following symmetric condition

$$
\begin{aligned}
& M\left(x_{11}, x_{12}, \ldots, x_{i 1}, x_{i 2}, \ldots, x_{n 1}, x_{n 2}\right) \\
& \quad=M\left(x_{11}, x_{12}, \ldots, x_{i 2}, x_{i 1}, \ldots, x_{n 1}, x_{n 2}\right)
\end{aligned}
$$

for all $x_{i j} \in V$, where $i \in\{1,2, \ldots, n\}$ and $j \in\{1,2\}$. Moreover, the mapping $M$ is given by

$$
\begin{aligned}
M & \left(x_{11}, x_{12}, x_{21}, x_{22}, \ldots, x_{n 1}, x_{n 2}\right) \\
& =\frac{1}{4^{n}} \sum_{i_{1}, \ldots, i_{n} \in\{1,-1\}}\left(i_{1} \cdots i_{n}\right) f\left(x_{11}+i_{1} x_{12}, \ldots, x_{n 1}+i_{n} x_{n 2}\right),
\end{aligned}
$$

where $x_{i j} \in V, i \in\{1,2, \ldots, n\}, j \in\{1,2\}$.

Proof. We prove this theorem by using induction on $n$. Clearly, Theorem 2 is true for $n=1$ thanks to Proposition 1 . Now, we assume that the present theorem is true for some $n \geq 2$, and we consider the case for $n+1$.
We first assume that there exists a multiadditive mapping $M: V^{2(n+1)} \rightarrow W$ such that

$$
\begin{aligned}
& f\left(x_{1}, x_{2}, \ldots, x_{n}, x_{n+1}\right) \\
& \quad=M\left(x_{1}, x_{1}, x_{2}, x_{2}, \ldots, x_{n}, x_{n}, x_{n+1}, x_{n+1}\right)
\end{aligned}
$$

for all $x_{1}, \ldots, x_{n}, x_{n+1} \in V$, and $M$ satisfies the following symmetric condition:

$$
\begin{aligned}
& M\left(x_{11}, x_{12}, \ldots, x_{i 1}, x_{i 2}, \ldots, x_{n 1}, x_{n 2}, x_{n+1,1}, x_{n+1,2}\right) \\
& \quad=M\left(x_{11}, x_{12}, \ldots, x_{i 2}, x_{i 1}, \ldots, x_{n 1}, x_{n 2}, x_{n+1,1}, x_{n+1,2}\right)
\end{aligned}
$$

for all $x_{i j} \in V$, where $i \in\{1,2, \ldots, n, n+1\}$ and $j \in\{1,2\}$. Then, for each $i \in\{1,2, \ldots, n, n+1\}$, we have that

$$
\begin{aligned}
& f\left(x_{1}, \ldots, x_{i-1}, x_{i}+x_{i}^{\prime}, x_{i+1}, \ldots, x_{n}, x_{n+1}\right) \\
&+f\left(x_{1}, \ldots, x_{i-1}, x_{i}-x_{i}^{\prime}, x_{i+1}, \ldots, x_{n}, x_{n+1}\right) \\
&= M\left(x_{1}, x_{1}, \ldots, x_{i-1}, x_{i-1}, x_{i}+x_{i}^{\prime}, x_{i}+x_{i}^{\prime}, x_{i+1},\right. \\
&\left.x_{i+1}, \ldots, x_{n}, x_{n}, x_{n+1}, x_{n+1}\right) \\
&+ M\left(x_{1}, x_{1}, \ldots, x_{i-1}, x_{i-1}, x_{i}-x_{i}^{\prime}, x_{i}-x_{i}^{\prime},\right. \\
&\left.x_{i+1}, x_{i+1}, \ldots, x_{n}, x_{n}, x_{n+1}, x_{n+1}\right) \\
&= 2 M\left(x_{1}, x_{1}, \ldots, x_{i-1}, x_{i-1}, x_{i}, x_{i}, x_{i+1}\right. \\
&\left.x_{i+1}, \ldots, x_{n}, x_{n}, x_{n+1}, x_{n+1}\right) \\
&+2 M\left(x_{1}, x_{1}, \ldots, x_{i-1}, x_{i-1}, x_{i}^{\prime}, x_{i}^{\prime}, x_{i+1},\right. \\
&\left.x_{i+1}, \ldots, x_{n}, x_{n}, x_{n+1}, x_{n+1}\right) \\
&= 2 f\left(x_{1}, \ldots, x_{i-1}, x_{i}, x_{i+1}, \ldots, x_{n}, x_{n+1}\right) \\
&+2 f\left(x_{1}, \ldots, x_{i-1}, x_{i}^{\prime}, x_{i+1}, \ldots, x_{n}, x_{n+1}\right)
\end{aligned}
$$

for all $x_{1}, x_{2}, \ldots, x_{n}, x_{n+1}, x_{i}^{\prime} \in V$. Thus, $f$ is multiquadratic.

Conversely, we assume that $f: V^{n+1} \rightarrow W$ is a multiquadratic function. We need to find the desired multiadditive function $M: V^{2(n+1)} \rightarrow W$. For this, we give the following notations. by

For each fixed $z \in V$, define the mapping $g_{z}: V^{n} \rightarrow W$

$$
g_{z}\left(x_{1}, \ldots, x_{n}\right):=f\left(x_{1}, \ldots, x_{n}, z\right) \quad \forall x_{1}, \ldots, x_{n} \in V .
$$

Then $g_{z}$ is a multiquadratic mapping (as $f: V^{n+1} \rightarrow W$ is multiquadratic). By induction, we let $M_{z}: V^{2 n} \rightarrow W$ denote the corresponding multiadditive mapping for $g_{z}$; that is, $M_{z}$ satisfies the symmetric condition (5) and

$$
g_{z}\left(x_{1}, \ldots, x_{n}\right)=M_{z}\left(x_{1}, x_{1}, x_{2}, x_{2}, \ldots, x_{n}, x_{n}\right)
$$


for all $x_{1}, \ldots, x_{n} \in V$. Moreover, the mapping $M_{z}: V^{2 n} \rightarrow$ $W$ is given by

$$
\begin{aligned}
M_{z} & \left(x_{11}, x_{12}, x_{21}, x_{22}, \ldots, x_{n 1}, x_{n 2}\right) \\
& =\frac{1}{4^{n}} \sum_{i_{1}, \ldots, i_{n} \in\{1,-1\}}\left(i_{1} \cdots i_{n}\right) g_{z}\left(x_{11}+i_{1} x_{12}, \ldots, x_{n 1}+i_{n} x_{n 2}\right)
\end{aligned}
$$

for all $x_{i j} \in V$, where $i \in\{1,2, \ldots, n\}$ and $j \in\{1,2\}$.

On the other hand, for any fixed elements $x_{11}, x_{12}, \ldots$, $x_{n 1}, x_{n 2} \in V$, define $h_{x_{11}, x_{12}, \ldots, x_{n 1}, x_{n 2}}: V \rightarrow W$ by

$$
h_{x_{11}, x_{12}, \ldots, x_{n 1}, x_{n 2}}(x)=M_{x}\left(x_{11}, x_{12}, \ldots, x_{n 1}, x_{n 2}\right)
$$

for all $x \in V$. It can be verified that $h_{x_{11}, x_{12}, \ldots, x_{n 1}, x_{n 2}}$ is a quadratic mapping. Thus, it follows from Proposition 1 that there exists a symmetric biadditive mapping $T_{x_{11}, x_{12}, \ldots, x_{n 1}, x_{n 2}}$ : $V \times V \rightarrow W$ such that

$$
h_{x_{11}, x_{12}, \ldots, x_{n 1}, x_{n 2}}(x)=T_{x_{11}, x_{12}, \ldots, x_{n 1}, x_{n 2}}(x, x)
$$

for all $x \in V$. The mapping $T_{x_{11}, x_{12}, \ldots, x_{n 1}, x_{n 2}}$ is given by

$$
\begin{aligned}
& T_{x_{11}, x_{12}, \ldots, x_{n 1}, x_{n 2}}(x, y) \\
& \quad=\frac{1}{4}\left[h_{x_{11}, x_{12}, \ldots, x_{n 1}, x_{n 2}}(x+y)-h_{x_{11}, x_{12}, \ldots, x_{n 1}, x_{n 2}}(x-y)\right]
\end{aligned}
$$

for all $x, y \in V$.

Now, we define the mapping $M: V^{2(n+1)} \rightarrow W$ by

$$
\begin{aligned}
& M\left(x_{11}, x_{12}, \ldots, x_{n 1}, x_{n 2}, x_{n+1,1}, x_{n+1,2}\right) \\
& :=\frac{1}{4}\left[M_{x_{n+1,1}+x_{n+1,2}}\left(x_{11}, x_{12}, \ldots, x_{n 1}, x_{n 2}\right)\right. \\
& \left.\quad-M_{x_{n+1,1}-x_{n+1,2}}\left(x_{11}, x_{12}, \ldots, x_{n 1}, x_{n 2}\right)\right]
\end{aligned}
$$

for all $x_{i j} \in V, i \in\{1,2, \ldots, n+1\}, j \in\{1,2\}$. In the following, we will show that $M$ is the desired function for $f$ : $V^{n+1} \rightarrow W$. First, we show that $M$ is multiadditive. Indeed, by the definition of $M$ (see (16)) and noting that for any $z \in V$ the function $M_{z}$ is multiadditive, one can obtain that for each $i \in\{1,2, \ldots, n\}$,

$$
\begin{gathered}
M\left(x_{11}, x_{12}, \ldots, x_{i 1}+x_{i 1}^{\prime}, x_{i 2}, \ldots, x_{n 1}, x_{n 2}, x_{n+1,1}, x_{n+1,2}\right) \\
=\frac{1}{4}\left[M_{x_{n+1,1}+x_{n+1,2}}\left(x_{11}, x_{12}, \ldots, x_{i 1}+x_{i 1}^{\prime}, x_{i 2}, \ldots, x_{n 1}, x_{n 2}\right)\right. \\
\quad-M_{x_{n+1,1}-x_{n+1,2}}\left(x_{11}, x_{12}, \ldots, x_{i 1}\right. \\
\left.\left.+x_{i 1}^{\prime}, x_{i 2}, \ldots, x_{n 1}, x_{n 2}\right)\right] \\
=\frac{1}{4}\left[M_{x_{n+1,1}+x_{n+1,2}}\left(x_{11}, x_{12}, \ldots, x_{i 1}, x_{i 2}, \ldots, x_{n 1}, x_{n 2}\right)\right. \\
\quad+M_{x_{n+1,1}+x_{n+1,2}}\left(x_{11}, x_{12}, \ldots, x_{i 1}^{\prime}, x_{i 2}, \ldots, x_{n 1}, x_{n 2}\right) \\
\quad-M_{x_{n+1,1}-x_{n+1,2}}\left(x_{11}, x_{12}, \ldots, x_{i 1}, x_{i 2}, \ldots, x_{n 1}, x_{n 2}\right) \\
\left.-M_{x_{n+1,1}-x_{n+1,2}}\left(x_{11}, x_{12}, \ldots, x_{i 1}^{\prime}, x_{i 2}, \ldots, x_{n 1}, x_{n 2}\right)\right]
\end{gathered}
$$

$$
\begin{aligned}
& =\frac{1}{4}\left[M_{x_{n+1,1}+x_{n+1,2}}\left(x_{11}, x_{12}, \ldots, x_{i 1}, x_{i 2}, \ldots, x_{n 1}, x_{n 2}\right)\right. \\
& \left.\quad-M_{x_{n+1,1}-x_{n+1,2}}\left(x_{11}, x_{12}, \ldots, x_{i 1}, x_{i 2}, \ldots, x_{n 1}, x_{n 2}\right)\right] \\
& +\frac{1}{4}\left[M_{x_{n+1,1}+x_{n+1,2}}\left(x_{11}, x_{12}, \ldots, x_{i 1}^{\prime}, x_{i 2}, \ldots, x_{n 1}, x_{n 2}\right)\right. \\
& \left.\quad-M_{x_{n+1,1}-x_{n+1,2}}\left(x_{11}, x_{12}, \ldots, x_{i 1}^{\prime}, x_{i 2}, \ldots, x_{n 1}, x_{n 2}\right)\right] \\
& =M\left(x_{11}, x_{12}, \ldots, x_{i 1}, x_{i 2}, \ldots, x_{n 1}, x_{n 2}, x_{n+1,1}, x_{n+1,2}\right) \\
& +M\left(x_{11}, x_{12}, \ldots, x_{i 1}^{\prime}, x_{i 2}, \ldots, x_{n 1}, x_{n 2}, x_{n+1,1}, x_{n+1,2}\right)
\end{aligned}
$$

for all $x_{i 1}^{\prime}, x_{11}, x_{12}, \ldots, x_{n+1,1}, x_{n+1,2} \in V$. Moreover, by the definition of $M$ in (16) and the notations we gave in (13) and (15), we have that

$$
\begin{aligned}
& M\left(x_{11}, x_{12}, \ldots, x_{n 1}, x_{n 2}, x_{n+1,1}+x_{n+1,1}^{\prime}, x_{n+1,2}\right) \\
& =\frac{1}{4}\left[M_{x_{n+1,1}+x_{n+1,1}^{\prime}+x_{n+1,2}}\left(x_{11}, x_{12}, \ldots, x_{n 1}, x_{n 2}\right)\right. \\
& \left.-M_{x_{n+1,1}+x_{n+1,1}^{\prime}-x_{n+1,2}}\left(x_{11}, x_{12}, \ldots, x_{n 1}, x_{n 2}\right)\right] \\
& =\frac{1}{4}\left[h_{x_{11}, x_{12}, \ldots, x_{n 1}, x_{n 2}}\left(x_{n+1,1}+x_{n+1,1}^{\prime}+x_{n+1,2}\right)\right. \\
& \left.-h_{x_{11}, x_{12}, \ldots, x_{n 1}, x_{n 2}}\left(x_{n+1,1}+x_{n+1,1}^{\prime}-x_{n+1,2}\right)\right] \\
& =T_{x_{11}, x_{12}, \ldots, x_{n 1}, x_{n 2}}\left(x_{n+1,1}+x_{n+1,1}^{\prime}, x_{n+1,2}\right) \\
& =T_{x_{11}, x_{12}, \ldots, x_{n 1}, x_{n 2}}\left(x_{n+1,1}, x_{n+1,2}\right) \\
& +T_{x_{11}, x_{12}, \ldots, x_{n 1}, x_{n 2}}\left(x_{n+1,1}^{\prime}, x_{n+1,2}\right) \\
& =\frac{1}{4}\left[h_{x_{11}, x_{12}, \ldots, x_{n 1}, x_{n 2}}\left(x_{n+1,1}+x_{n+1,2}\right)\right. \\
& \left.-h_{x_{11}, x_{12}, \ldots, x_{n 1}, x_{n 2}}\left(x_{n+1,1}-x_{n+1,2}\right)\right] \\
& +\frac{1}{4}\left[h_{x_{11}, x_{12}, \ldots, x_{n 1}, x_{n 2}}\left(x_{n+1,1}^{\prime}+x_{n+1,2}\right)\right. \\
& \left.-h_{x_{11}, x_{12}, \ldots, x_{n 1}, x_{n 2}}\left(x_{n+1,1}^{\prime}-x_{n+1,2}\right)\right] \\
& =\frac{1}{4}\left[M_{x_{n+1,1}+x_{n+1,2}}\left(x_{11}, x_{12}, \ldots, x_{n 1}, x_{n 2}\right)\right. \\
& \left.-M_{x_{n+1,1}-x_{n+1,2}}\left(x_{11}, x_{12}, \ldots, x_{n 1}, x_{n 2}\right)\right] \\
& +\frac{1}{4}\left[M_{x_{n+1,1}^{\prime}+x_{n+1,2}}\left(x_{11}, x_{12}, \ldots, x_{n 1}, x_{n 2}\right)\right. \\
& \left.-M_{x_{n+1,1}^{\prime}-x_{n+1,2}}\left(x_{11}, x_{12}, \ldots, x_{n 1}, x_{n 2}\right)\right] \\
& =M\left(x_{11}, x_{12}, \ldots, x_{n 1}, x_{n 2}, x_{n+1,1}, x_{n+1,2}\right) \\
& +M\left(x_{11}, x_{12}, \ldots, x_{n 1}, x_{n 2}, x_{n+1,1}^{\prime}, x_{n+1,2}\right)
\end{aligned}
$$


for all $x_{n+1,1}^{\prime}, x_{11}, x_{12}, \ldots, x_{n 1}, x_{n 2}, x_{n+1,1}, x_{n+1,2} \in V$. Similarly, we can see that $M$ is additive in the other variables. Thus, we have shown that $M$ is multiadditive.

Furthermore, since $f$ is multiquadratic, we obtain that

$$
\begin{gathered}
f\left(x_{1}, x_{2}, \ldots, x_{n}, 0\right)=0 \\
f\left(x_{1}, \ldots, x_{n}, 2 x_{n+1}\right)=4 f\left(x_{1}, \ldots, x_{n}, x_{n+1}\right)
\end{gathered}
$$

for all $x_{1}, \ldots, x_{n}, x_{n+1} \in V$. Thus, by the definition of $M$ in (16) and the notations we gave in (10) and (11), one has

$$
\begin{aligned}
& f\left(x_{1}, x_{2}, \ldots, x_{n}, x_{n+1}\right) \\
& =\frac{1}{4}\left[f\left(x_{1}, x_{2}, \ldots, x_{n}, 2 x_{n+1}\right)-f\left(x_{1}, x_{2}, \ldots, x_{n}, 0\right)\right] \\
& =\frac{1}{4}\left[g_{2 x_{n+1}}\left(x_{1}, x_{2}, \ldots, x_{n}\right)-g_{0}\left(x_{1}, x_{2}, \ldots, x_{n}\right)\right] \\
& =\frac{1}{4}\left[M_{2 x_{n+1}}\left(x_{1}, x_{1}, x_{2}, x_{2}, \ldots, x_{n}, x_{n}\right)\right. \\
& \left.\quad-M_{0}\left(x_{1}, x_{1}, x_{2}, x_{2}, \ldots, x_{n}, x_{n}\right)\right] \\
& =M\left(x_{1}, x_{1}, x_{2}, x_{2}, \ldots, x_{n}, x_{n}, x_{n+1}, x_{n+1}\right)
\end{aligned}
$$

for all $x_{1}, \ldots, x_{n}, x_{n+1} \in V$.

Now, we verify the expression of the mapping $M$. By the definition of $M$ again and the notations we gave in (10) and (12), also noting that $f$ is multiquadratic, one can obtain that

$$
\begin{aligned}
& M\left(x_{11}, x_{12}, \ldots, x_{n 1}, x_{n 2}, x_{n+1,1}, x_{n+1,2}\right) \\
& =\frac{1}{4}\left[M_{x_{n+1,1}+x_{n+1,2}}\left(x_{11}, x_{12}, \ldots, x_{n 1}, x_{n 2}\right)\right. \\
& \left.-M_{x_{n+1,1}-x_{n+1,2}}\left(x_{11}, x_{12}, \ldots, x_{n 1}, x_{n 2}\right)\right] \\
& =\frac{1}{4}\left[\frac { 1 } { 4 ^ { n } } \sum _ { i _ { 1 } , \ldots , i _ { n } \in \{ 1 , - 1 \} } ( i _ { 1 } \cdots i _ { n } ) g _ { x _ { n + 1 , 1 } + x _ { n + 1 , 2 } } \left(x_{11}+i_{1} x_{12}, \ldots\right.\right. \text {, } \\
& \left.x_{n 1}+i_{n} x_{n 2}\right) \\
& -\frac{1}{4^{n}} \sum_{i_{1}, \ldots, i_{n} \in\{1,-1\}}\left(i_{1} \cdots i_{n}\right) g_{x_{n+1,1}-x_{n+1,2}}\left(x_{11}+i_{1} x_{12}, \ldots,\right. \\
& \left.\left.x_{n 1}+i_{n} x_{n 2}\right)\right] \\
& =\frac{1}{4^{n+1}}\left[\sum_{i_{1}, \ldots, i_{n} \in\{1,-1\}}\left(i_{1} \cdots i_{n}\right)\right. \\
& \times f\left(x_{11}+i_{1} x_{12}, \ldots, x_{n 1}\right. \\
& \left.+i_{n} x_{n 2}, x_{n+1,1}+x_{n+1,2}\right)
\end{aligned}
$$

$$
\begin{aligned}
& -\sum_{i_{1}, \ldots, i_{n} \in\{1,-1\}}\left(i_{1} \cdots i_{n}\right) \\
& \times f\left(x_{11}+i_{1} x_{12}, \ldots, x_{n 1}\right. \\
& \left.\left.+i_{n} x_{n 2}, x_{n+1,1}-x_{n+1,2}\right)\right] \\
& =\frac{1}{4^{n+1}} \sum_{i_{1}, \ldots, i_{n}, i_{n+1} \in\{1,-1\}}\left(i_{1} \cdots i_{n} \cdot i_{n+1}\right) \\
& \times f\left(x_{11}+i_{1} x_{12}, \ldots, x_{n 1}\right. \\
& \left.+i_{n} x_{n 2}, x_{n+1,1}+i_{n+1} x_{n+1,2}\right)
\end{aligned}
$$

for all $x_{i j} \in V, i \in\{1,2, \ldots, n+1\}, j \in\{1,2\}$.

Finally, we check the symmetric property of $M$. Fix any $x_{i j} \in V$, where $i \in\{1,2, \ldots, n+1\}$ and $j \in\{1,2\}$. Since $f$ is multiquadratic, it follows that $f$ is an even mapping in each variable. Then by (21), it is easy to verify that

$$
\begin{aligned}
& M\left(x_{11}, x_{12}, \ldots, x_{n 1}, x_{n 2}, x_{n+1,1}, x_{n+1,2}\right) \\
& \quad=M\left(x_{11}, x_{12}, \ldots, x_{n 1}, x_{n 2}, x_{n+1,2}, x_{n+1,1}\right) .
\end{aligned}
$$

Moreover, due to the symmetric property of $M_{x_{n+1,1}+x_{n+1,2}}$ and $M_{x_{n+1,1}-x_{n+1,2}}$ and from the definition of $M$ (see (16)) we can get

$$
\begin{aligned}
& M\left(x_{11}, x_{12}, \ldots, x_{i 1}, x_{i 2}, \ldots, x_{n 1}, x_{n 2}, x_{n+1,1}, x_{n+1,2}\right) \\
& \quad=M\left(x_{11}, x_{12}, \ldots, x_{i 2}, x_{i 1}, \ldots, x_{n 1}, x_{n 2}, x_{n+1,1}, x_{n+1,2}\right)
\end{aligned}
$$

for each $i \in\{1,2, \ldots, n\}$. So the desired symmetric property of $M$ is proved. Thus, we have shown that $M: V^{2(n+1)} \rightarrow W$ is the desired multiadditive mapping for the multiquadratic mapping $f: V^{n} \rightarrow W$. The proof is complete.

\section{A Characterization for the Multiquadratic Functional Equation}

The following theorem provides a sufficient and necessary condition for a mapping to be multiquadratic.

Theorem 3. Let $V$ be a commutative semigroup with the identity element 0 , and let $W$ be a linear space. A mapping $f$ : $V^{n} \rightarrow W$ is multiquadratic if and only if

$$
\begin{gathered}
\sum_{i_{1}, \ldots, i_{n} \in\{1,-1\}} f\left(x_{11}+i_{1} x_{12}, \ldots, x_{n 1}+i_{n} x_{n 2}\right) \\
=2^{n} \sum_{j_{1}, \ldots, j_{n} \in\{1,2\}} f\left(x_{1 j_{1}}, \ldots, x_{n j_{n}}\right)
\end{gathered}
$$

for all $\left(x_{11}, \ldots, x_{n 1}\right),\left(x_{12}, \ldots, x_{n 2}\right) \in V^{n}$.

Proof. Assume that $f: V^{n} \rightarrow W$ satisfies (24). Putting

$$
\left(x_{11}, \ldots, x_{n 1}\right)=\left(x_{12}, \ldots, x_{n 2}\right)=(0, \ldots, 0)
$$


in (24) we get $2^{n} f(0, \ldots, 0)=2^{2 n} f(0, \ldots, 0)$, and consequently, we have $f(0, \ldots, 0)=0$. Next, fix $j \in\{1, \ldots, n\}, x_{j 1} \in$ $V$, and put $x_{j 2}=x_{k i_{k}}=0$, where $i_{k} \in\{1,2\}$, for $k \in\{1, \ldots, n\} \backslash$ $\{j\}$. Then, by (24),

$$
\begin{aligned}
2^{n} f( & \left(0, \ldots, 0, x_{j 1}, 0, \ldots, 0\right) \\
& =2^{n} 2^{n-1} f\left(0, \ldots, 0, x_{j 1}, 0, \ldots, 0\right),
\end{aligned}
$$

and thus $f\left(0, \ldots, 0, x_{j 1}, 0, \ldots, 0\right)=0$. Continuing in this fashion, we obtain that $f(x)=0$ for any $x \in V^{n}$ with at least one component which is equal to 0 .

Now, fix $j \in\{1, \ldots, n\}, x_{11}, \ldots, x_{n 1}, x_{j 2} \in V$ and put $x_{k 2}=$ 0 for $k \in\{1, \ldots, n\} \backslash\{j\}$ in (24). Then

$$
\begin{aligned}
2^{n-1} f\left(x_{11}, \ldots, x_{j 1}+x_{j 2}, \ldots, x_{n 1}\right) \\
+2^{n-1} f\left(x_{11}, \ldots, x_{j 1}-x_{j 2}, \ldots, x_{n 1}\right) \\
=2^{n}\left[f\left(x_{11}, \ldots, x_{j 1}, \ldots, x_{n 1}\right)\right. \\
\left.+f\left(x_{11}, \ldots, x_{j 2}, \ldots, x_{n 1}\right)\right],
\end{aligned}
$$

and thus

$$
\begin{aligned}
f\left(x_{11}, \ldots, x_{j 1}+x_{j 2}, \ldots, x_{n 1}\right) \\
\quad+f\left(x_{11}, \ldots, x_{j 1}-x_{j 2}, \ldots, x_{n 1}\right) \\
=2 f\left(x_{11}, \ldots, x_{j 1}, \ldots, x_{n 1}\right) \\
\quad+2 f\left(x_{11}, \ldots, x_{j 2}, \ldots, x_{n 1}\right),
\end{aligned}
$$

which proves that $f$ is multiquadratic.

Conversely, we assume that $f$ is multiquadratic, and we prove (24) by mathematical induction. If $f: V \rightarrow W$ is quadratic, then $f\left(x_{11}+x_{12}\right)+f\left(x_{11}-x_{12}\right)=2 f\left(x_{11}\right)+2 f\left(x_{12}\right)$ for all $x_{11}, x_{12} \in V$. So, (24) holds for $n=1$. It is easy to verify that (24) holds for $n=2$. Indeed,

$$
\begin{aligned}
& f\left(x_{11}+x_{12}, x_{21}+x_{22}\right) \\
& +f\left(x_{11}+x_{12}, x_{21}-x_{22}\right)+f\left(x_{11}-x_{12}, x_{21}+x_{22}\right) \\
& +f\left(x_{11}-x_{12}, x_{21}-x_{22}\right) \\
& =2 f\left(x_{11}+x_{12}, x_{21}\right)+2 f\left(x_{11}+x_{12}, x_{22}\right) \\
& \quad+2 f\left(x_{11}-x_{12}, x_{21}\right)+2 f\left(x_{11}-x_{12}, x_{22}\right) \\
& =4\left[f\left(x_{11}, x_{21}\right)+f\left(x_{12}, x_{21}\right)\right. \\
& \left.\quad+f\left(x_{11}, x_{22}\right)+f\left(x_{12}, x_{22}\right)\right]
\end{aligned}
$$

for all $x_{11}, x_{12}, x_{21}, x_{22} \in V$. Assume that (24) holds for some positive integer $n>2$. Then,

$$
\begin{gathered}
\sum_{i_{1}, \ldots, i_{n+1} \in\{1,-1\}} f\left(x_{11}+i_{1} x_{12}, \ldots, x_{n 1}\right. \\
\left.+i_{n} x_{n 2}, x_{n+1,1}+i_{n+1} x_{n+1,2}\right) \\
=\sum_{i_{1}, \ldots, i_{n} \in\{1,-1\}}\left[f \left(x_{11}+i_{1} x_{12}, \ldots, x_{n 1}\right.\right. \\
\left.+i_{n} x_{n 2}, x_{n+1,1}+x_{n+1,2}\right) \\
+f\left(x_{11}+i_{1} x_{12}, \ldots, x_{n 1}\right. \\
\left.\left.+i_{n} x_{n 2}, x_{n+1,1}-x_{n+1,2}\right)\right] \\
=2^{n} \sum_{j_{1}, \ldots, j_{n} \in\{1,2\}}\left[f\left(x_{1 j_{1}}, \ldots, x_{n j_{n}}, x_{n+1,1}+x_{n+1,2}\right)\right. \\
\left.+f\left(x_{1 j_{1}}, \ldots, x_{n j_{n}}, x_{n+1,1}-x_{n+1,2}\right)\right] \\
=2^{n} \sum_{j_{1}, \ldots, j_{n} \in\{1,2\}}\left[\begin{array}{l}
2 f\left(x_{1 j_{1}}, \ldots, x_{n j_{n}}, x_{n+1,1}\right) \\
\left.+2 f\left(x_{1 j_{1}}, \ldots, x_{n j_{n}}, x_{n+1,2}\right)\right] \\
\sum_{j_{1}, \ldots, j_{n}, j_{n+1} \in\{1,2\}} f\left(x_{1 j_{1}}, \ldots, x_{n j_{n}}, x_{n+1 j_{n+1}}\right) .
\end{array}\right.
\end{gathered}
$$

Thus, (24) holds for $n+1$, and this completes the proof.

\section{Stability}

In this section, we give two results on the stability of the multiquadratic functional equation. Throughout this section, let $V$ be a commutative semigroup with the identity element 0 , and let $W$ be a Banach space.

Theorem 4. Assume that for every $i \in\{1, \ldots, n\}, \varphi_{i}: V^{n+1} \rightarrow$ $[0, \infty)$ is a mapping such that for any $\left(x_{1}, \ldots, x_{n+1}\right) \in V^{n+1}$

$$
\begin{aligned}
\widetilde{\varphi}_{i}\left(x_{1}, \ldots, x_{n+1}\right) & \\
:=\sum_{j=0}^{\infty} \frac{1}{4^{j}}[ & \varphi_{i}\left(2^{j} x_{1}, x_{2}, \ldots, x_{n+1}\right) \\
& +\cdots+\varphi_{i}\left(x_{1}, \ldots, x_{i-2}, 2^{j} x_{i-1}, x_{i}, \ldots, x_{n+1}\right) \\
& +\frac{1}{4} \varphi_{i}\left(x_{1}, \ldots, x_{i-1}, 2^{j} x_{i}, 2^{j} x_{i+1}, x_{i+2}, \ldots, x_{n+1}\right) \\
& +\varphi_{i}\left(x_{1}, \ldots, x_{i}, x_{i+1}, 2^{j} x_{i+2}, x_{i+3}, \ldots, x_{n+1}\right) \\
& \left.+\cdots+\varphi_{i}\left(x_{1}, \ldots, x_{n}, 2^{j} x_{n+1}\right)\right]<\infty .
\end{aligned}
$$


If $f: V^{n} \rightarrow W$ is a function satisfying

$$
\begin{gathered}
\| f\left(x_{1}, \ldots, x_{i-1}, x_{i}+x_{i}^{\prime}, x_{i+1}, \ldots, x_{n}\right) \\
+f\left(x_{1}, \ldots, x_{i-1}, x_{i}-x_{i}^{\prime}, x_{i+1}, \ldots, x_{n}\right) \\
-2 f\left(x_{1}, \ldots, x_{i-1}, x_{i}, x_{i+1}, \ldots, x_{n}\right) \\
-2 f\left(x_{1}, \ldots, x_{i-1}, x_{i}^{\prime}, x_{i+1}, \ldots, x_{n}\right) \| \\
\leq \varphi_{i}\left(x_{1}, \ldots, x_{i}, x_{i}^{\prime}, x_{i+1}, \ldots, x_{n}\right)
\end{gathered}
$$

for all $\left(x_{1}, \ldots, x_{i}, x_{i}^{\prime}, x_{i+1}, \ldots, x_{n}\right) \in V^{n+1}, i \in\{1, \ldots, n\}$, then for every $i \in\{1, \ldots, n\}$ there exists a multiquadratic mapping $F_{i}: V^{n} \rightarrow W$ such that for any $\left(x_{1}, \ldots, x_{n}\right) \in V^{n}$ one has

$$
\begin{aligned}
& \| f\left(x_{1}, \ldots, x_{n}\right)-\frac{1}{3} f\left(x_{1}, \ldots, x_{i-1}, 0, x_{i+1}, \ldots, x_{n}\right) \\
& -F_{i}\left(x_{1}, \ldots, x_{n}\right) \| \\
& \quad \leq \widetilde{\varphi}_{i}\left(x_{1}, \ldots, x_{i}, x_{i}, x_{i+1}, \ldots, x_{n}\right) .
\end{aligned}
$$

For every $i \in\{1, \ldots, n\}$ the function $F_{i}$ is given by

$$
F_{i}\left(x_{1}, \ldots, x_{n}\right):=\lim _{j \rightarrow \infty} \frac{1}{4^{j}} f\left(x_{1}, \ldots, x_{i-1}, 2^{j} x_{i}, x_{i+1}, \ldots, x_{n}\right)
$$

for all $\left(x_{1}, \ldots, x_{n}\right) \in V^{n}$.

Proof. Fix $x_{1}, \ldots, x_{n} \in V, j \in N \cup\{0\}$ (where $N$ denotes the set of the positive integers) and $i \in\{1, \ldots, n\}$. Putting $x_{i}^{\prime}:=x_{i}$ in (32), we get

$$
\begin{aligned}
& \| f\left(x_{1}, \ldots, x_{i-1}, 2 x_{i}, x_{i+1}, \ldots, x_{n}\right) \\
& \quad+f\left(x_{1}, \ldots, x_{i-1}, 0, x_{i+1}, \ldots, x_{n}\right)-4 f\left(x_{1}, \ldots, x_{n}\right) \| \\
& \quad \leq \varphi_{i}\left(x_{1}, \ldots, x_{i}, x_{i}, x_{i+1}, \ldots, x_{n}\right) .
\end{aligned}
$$

Hence

$$
\begin{aligned}
& \| f\left(x_{1}, \ldots, x_{n}\right)-\frac{1}{4} f\left(x_{1}, \ldots, x_{i-1}, 2 x_{i}, x_{i+1}, \ldots, x_{n}\right) \\
& -\frac{1}{4} f\left(x_{1}, \ldots, x_{i-1}, 0, x_{i+1}, \ldots, x_{n}\right) \| \\
& \quad \leq \frac{1}{4} \varphi_{i}\left(x_{1}, \ldots, x_{i}, x_{i}, x_{i+1}, \ldots, x_{n}\right) .
\end{aligned}
$$

Dividing both sides of the above inequality by $4^{j}$ and replacing $x_{i}$ by $2^{j} x_{i}$, we obtain

$$
\begin{aligned}
& \| \frac{1}{4^{j}} f\left(x_{1}, \ldots, x_{i-1}, 2^{j} x_{i}, x_{i+1}, \ldots, x_{n}\right) \\
& -\frac{1}{4^{j+1}} f\left(x_{1}, \ldots, x_{i-1}, 2^{j+1} x_{i}, x_{i+1}, \ldots, x_{n}\right) \\
& \quad-\frac{1}{4^{j+1}} f\left(x_{1}, \ldots, x_{i-1}, 0, x_{i+1}, \ldots, x_{n}\right) \| \\
& \quad \leq \frac{1}{4^{j+1}} \varphi_{i}\left(x_{1}, \ldots, 2^{j} x_{i}, 2^{j} x_{i}, x_{i+1}, \ldots, x_{n}\right),
\end{aligned}
$$

and consequently for any nonnegative integers $l$ and $m$ with $l<m$, we obtain

$$
\begin{aligned}
& \| \frac{1}{4^{l}} f\left(x_{1}, \ldots, x_{i-1}, 2^{l} x_{i}, x_{i+1}, \ldots, x_{n}\right) \\
& -\frac{1}{4^{m}} f\left(x_{1}, \ldots, x_{i-1}, 2^{m} x_{i}, x_{i+1}, \ldots, x_{n}\right) \\
& \quad-\sum_{j=l}^{m-1} \frac{1}{4^{j+1}} f\left(x_{1}, \ldots, x_{i-1}, 0, x_{i+1}, \ldots, x_{n}\right) \| \\
& \quad \leq \sum_{j=l}^{m-1} \frac{1}{4^{j+1}} \varphi_{i}\left(x_{1}, \ldots, 2^{j} x_{i}, 2^{j} x_{i}, x_{i+1}, \ldots, x_{n}\right) .
\end{aligned}
$$

Therefore, it follows from (31) that $\left\{1 / 4^{j} f\left(x_{1}, \ldots\right.\right.$, $\left.\left.x_{i-1}, 2^{j} x_{i}, x_{i+1}, \ldots, x_{n}\right)\right\}_{j \in N}$ is a Cauchy sequence. Since the space $W$ is complete, this sequence is convergent, and we define $F_{i}: V^{n} \rightarrow W$ by (34). Putting $l=0$, letting $m \rightarrow \infty$ in (38), and using (31), we see that (33) holds.

Finally, fix also $x_{i}^{\prime} \in V, j \in N$, and notice that according to (32) we have

$$
\begin{aligned}
& \| \frac{1}{4^{j}} f\left(x_{1}, \ldots, x_{i-1}, 2^{j}\left(x_{i}+x_{i}^{\prime}\right), x_{i+1}, \ldots, x_{n}\right) \\
& +\frac{1}{4^{j}} f\left(x_{1}, \ldots, x_{i-1}, 2^{j}\left(x_{i}-x_{i}^{\prime}\right), x_{i+1}, \ldots, x_{n}\right) \\
& \quad-\frac{2}{4^{j}} f\left(x_{1}, \ldots, x_{i-1}, 2^{j} x_{i}, x_{i+1}, \ldots, x_{n}\right) \\
& -\frac{2}{4^{j}} f\left(x_{1}, \ldots, x_{i-1}, 2^{j} x_{i}^{\prime}, x_{i+1}, \ldots, x_{n}\right) \| \\
& \quad \leq \frac{1}{4^{j}} \varphi_{i}\left(x_{1}, \ldots, 2^{j} x_{i}, 2^{j} x_{i}^{\prime}, x_{i+1}, \ldots, x_{n}\right) .
\end{aligned}
$$

Next, fix $k \in\{1, \ldots, n\} \backslash\{i\}, x_{k}^{\prime} \in V$, and assume that $k<i$ (the same arguments apply to the case where $k>i$ ). Then, it follows from (32) that

$$
\begin{aligned}
& \| \frac{1}{4^{j}} f\left(x_{1}, \ldots, x_{k-1}, x_{k}\right. \\
& \left.\quad+x_{k}^{\prime}, x_{k+1}, \ldots, x_{i-1}, 2^{j} x_{i}, x_{i+1}, \ldots, x_{n}\right) \\
& +\frac{1}{4^{j}} f\left(x_{1}, \ldots, x_{k-1}, x_{k}\right. \\
& \left.\quad-x_{k}^{\prime}, x_{k+1}, \ldots, x_{i-1}, 2^{j} x_{i}, x_{i+1}, \ldots, x_{n}\right) \\
& -\frac{2}{4^{j}} f\left(x_{1}, \ldots, x_{i-1}, 2^{j} x_{i}, x_{i+1}, \ldots, x_{n}\right) \\
& -\frac{2}{4^{j}} f\left(x_{1}, \ldots, x_{k-1}, x_{k}^{\prime}, x_{k+1}, \ldots,\right. \\
& \left.\quad x_{i-1}, 2^{j} x_{i}, x_{i+1}, \ldots, x_{n}\right) \| \\
& \leq \frac{1}{4^{j}} \varphi_{k}\left(x_{1}, \ldots, x_{k}, x_{k}^{\prime}, x_{k+1}, \ldots, x_{i-1}, 2^{j} x_{i}, x_{i+1}, \ldots, x_{n}\right) .
\end{aligned}
$$


Letting $j \rightarrow \infty$ in the above two inequalities and using (31), we see that the mapping $F_{i}$ is multiquadratic.

Theorem 5. Assume that $\varphi: V^{2 n} \rightarrow[0, \infty)$ is a mapping such that

$$
\begin{aligned}
& \widetilde{\varphi}\left(x_{11}, x_{12}, \ldots, x_{n 1}, x_{n 2}\right) \\
& \quad:=\sum_{j=0}^{\infty} \frac{1}{4^{n(j+1)}} \varphi\left(2^{j} x_{11}, 2^{j} x_{12}, \ldots, 2^{j} x_{n 1}, 2^{j} x_{n 2}\right)<\infty
\end{aligned}
$$

for all $\left(x_{11}, x_{12}, \ldots, x_{n 1}, x_{n 2}\right) \in V^{2 n}$. If $f: V^{n} \rightarrow W$ is a function satisfying

$$
\begin{aligned}
& \| \sum_{i_{1}, \ldots, i_{n} \in\{1,-1\}} f\left(x_{11}+i_{1} x_{12}, \ldots, x_{n 1}+i_{n} x_{n 2}\right) \\
& -2^{n} \sum_{j_{1}, \ldots, j_{n} \in\{1,2\}} f\left(x_{1 j_{1}}, \ldots, x_{n j_{n}}\right) \| \\
& \leq \varphi\left(x_{11}, x_{12}, \ldots, x_{n 1}, x_{n 2}\right)
\end{aligned}
$$

for all $\left(x_{11}, x_{12}, \ldots, x_{n 1}, x_{n 2}\right) \in V^{2 n}$ and letting $f(x)=0$ for any $x \in V^{n}$ with one component which is equal to 0 , then there exists a unique multiquadratic mapping $F: V^{n} \rightarrow W$ such that

$$
\begin{gathered}
\left\|f\left(x_{11}, \ldots, x_{n 1}\right)-F\left(x_{11}, \ldots, x_{n 1}\right)\right\| \\
\leq \widetilde{\varphi}\left(x_{11}, x_{11}, \ldots, x_{n 1}, x_{n 1}\right)
\end{gathered}
$$

for all $\left(x_{11}, \ldots, x_{n 1}\right) \in V^{n}$. The function $F$ is given by

$$
F\left(x_{11}, \ldots, x_{n 1}\right):=\lim _{j \rightarrow \infty} \frac{1}{4^{n j}} f\left(2^{j} x_{11}, \ldots, 2^{j} x_{n 1}\right)
$$

for all $\left(x_{11}, \ldots, x_{n 1}\right) \in V^{n}$.

Proof. Fix $\left(x_{11}, \ldots, x_{n 1}\right) \in V^{n}$ and $j \in N \cup\{0\}$. Putting $x_{i 2}:=$ $x_{i 1}$ for $i \in\{1, \ldots, n\}$ in (42), we get

$$
\begin{aligned}
& \left\|f\left(2 x_{11}, \ldots, 2 x_{n 1}\right)-4^{n} f\left(x_{11}, \ldots, x_{n 1}\right)\right\| \\
& \leq \varphi\left(x_{11}, x_{11}, \ldots, x_{n 1}, x_{n 1}\right) .
\end{aligned}
$$

Dividing both sides of the above inequality by $4^{n(j+1)}$ and replacing $x_{i 1}$ by $2^{j} x_{i 1}$ for $i \in\{1, \ldots, n\}$, we see that

$$
\begin{aligned}
& \left\|\frac{1}{4^{n(j+1)}} f\left(2^{j+1} x_{11}, \ldots, 2^{j+1} x_{n 1}\right)-\frac{1}{4^{n j}} f\left(2^{j} x_{11}, \ldots, 2^{j} x_{n 1}\right)\right\| \\
& \quad \leq \frac{1}{4^{n(j+1)}} \varphi\left(2^{j} x_{11}, 2^{j} x_{11}, \ldots, 2^{j} x_{n 1}, 2^{j} x_{n 1}\right),
\end{aligned}
$$

and consequently for any nonnegative integers $l$ and $m$ with $l<m$ we obtain

$$
\begin{gathered}
\left\|\frac{1}{4^{n m}} f\left(2^{m} x_{11}, \ldots, 2^{m} x_{n 1}\right)-\frac{1}{4^{n l}} f\left(2^{l} x_{11}, \ldots, 2^{l} x_{n 1}\right)\right\| \\
\leq \sum_{j=l}^{m-1} \frac{1}{4^{n(j+1)}} \varphi\left(2^{j} x_{11}, 2^{j} x_{11}, \ldots, 2^{j} x_{n 1}, 2^{j} x_{n 1}\right) .
\end{gathered}
$$

Therefore, it follows from (41) that $\left\{1 / 4^{n j} f\left(2^{j} x_{11}, \ldots\right.\right.$, $\left.\left.2^{j} x_{n 1}\right)\right\}_{j \in N}$ is a Cauchy sequence. Since the space $W$ is complete, this sequence is convergent, and we define $F$ : $V^{n} \rightarrow W$ by (44). Putting $l=0$, taking $m \rightarrow \infty$ in (47), and using (41), we can see that the inequality (43) holds.

Next, fix also $\left(x_{12}, \ldots, x_{n 2}\right) \in V^{n}$, and note that according to (42) we have

$$
\begin{aligned}
& \| \frac{1}{4^{n j}} \sum_{i_{1}, \ldots, i_{n} \in\{1,-1\}} f\left(2^{j}\left(x_{11}+i_{1} x_{12}\right), \ldots, 2^{j}\left(x_{n 1}+i_{n} x_{n 2}\right)\right) \\
& -2^{n} \sum_{j_{1}, \ldots, j_{n} \in\{1,2\}} \frac{1}{4^{n j}} f\left(2^{j} x_{1 j_{1}}, \ldots, 2^{j} x_{n j_{n}}\right) \| \\
& \quad \leq \frac{1}{4^{n j}} \varphi\left(2^{j} x_{11}, 2^{j} x_{12}, \ldots, 2^{j} x_{n 1}, 2^{j} x_{n 2}\right) .
\end{aligned}
$$

Letting $j \rightarrow \infty$ in the above inequality and using (41), we see that $F$ satisfies (24). By Theorem 3, we obtain that $F$ is multiquadratic.

Finally, assume that $F^{\prime}: V^{n} \rightarrow W$ is another multiquadratic mapping satisfying (43). Fix $k \in N \cup\{0\}$. Since $F$ and $F^{\prime}$ are multiquadratic mappings, it is easy to verify that

$$
\begin{aligned}
F\left(2^{k} x_{11}, \ldots, 2^{k} x_{n 1}\right) & =4^{n k} F\left(x_{11}, \ldots, x_{n 1}\right), \\
F^{\prime}\left(2^{k} x_{11}, \ldots, 2^{k} x_{n 1}\right) & =4^{n k} F^{\prime}\left(x_{11}, \ldots, x_{n 1}\right) .
\end{aligned}
$$

Then, using (41) and (43), we have

$$
\begin{gathered}
\left\|F\left(x_{11}, \ldots, x_{n 1}\right)-F^{\prime}\left(x_{11}, \ldots, x_{n 1}\right)\right\| \\
=\| \frac{1}{4^{n k}} F\left(2^{k} x_{11}, \ldots, 2^{k} x_{n 1}\right) \\
\quad-\frac{1}{4^{n k}} F^{\prime}\left(2^{k} x_{11}, \ldots, 2^{k} x_{n 1}\right) \| \\
\leq \| \frac{1}{4^{n k}} F\left(2^{k} x_{11}, \ldots, 2^{k} x_{n 1}\right) \\
\quad-\frac{1}{4^{n k}} f\left(2^{k} x_{11}, \ldots, 2^{k} x_{n 1}\right) \| \\
+\| \frac{1}{4^{n k}} f\left(2^{k} x_{11}, \ldots, 2^{k} x_{n 1}\right) \\
\quad-\frac{1}{4^{n k}} F^{\prime}\left(2^{k} x_{11}, \ldots, 2^{k} x_{n 1}\right) \|
\end{gathered}
$$




$$
\begin{aligned}
& \leq \frac{2}{4^{n k}} \widetilde{\varphi}\left(2^{k} x_{11}, 2^{k} x_{11}, \ldots, 2^{k} x_{n 1}, 2^{k} x_{n 1}\right) \\
& =2 \sum_{j=k}^{\infty} \frac{1}{4^{n(j+1)}} \varphi\left(2^{j} x_{11}, 2^{j} x_{11}, \ldots, 2^{j} x_{n 1}, 2^{j} x_{n 1}\right) ;
\end{aligned}
$$

hence letting $k \rightarrow \infty$ we obtain $F=F^{\prime}$.

\section{Conflict of Interests}

The authors declare that there is no conflict of interests regarding the publication of this paper.

\section{References}

[1] S. M. Ulam, A Collection of Mathematical Problems, Interscience Publishers, New York, NY, USA, 1960.

[2] D. H. Hyers, "On the stability of the linear functional equation," Proceedings of the National Academy of Sciences of the United States of America, vol. 27, no. 4, pp. 222-224, 1941.

[3] Th. M. Rassias, "On the stability of the linear mapping in Banach spaces," Proceedings of the American Mathematical Society, vol. 72, no. 2, pp. 297-300, 1978.

[4] S.-M. Jung, "Hyers-Ulam-Rassias stability of Jensen's equation and its application," Proceedings of the American Mathematical Society, vol. 126, no. 11, pp. 3137-3143, 1998.

[5] K.-W. Jun and H.-M. Kim, "The generalized Hyers-UlamRassias stability of a cubic functional equation," Journal of Mathematical Analysis and Applications, vol. 274, no. 2, pp. 867878, 2002.

[6] K.-W. Jun and H.-M. Kim, "Stability problem of Ulam for generalized forms of Cauchy functional equation," Journal of Mathematical Analysis and Applications, vol. 312, no. 2, pp. 535547, 2005.

[7] G. H. Kim, "On the stability of the Pexiderized trigonometric functional equation," Applied Mathematics and Computation, vol. 203, no. 1, pp. 99-105, 2008.

[8] S. H. Lee, S. M. Im, and I. S. Hwang, "Quartic functional equations," Journal of Mathematical Analysis and Applications, vol. 307, no. 2, pp. 387-394, 2005.

[9] A. Najati and Th. M. Rassias, "Stability of a mixed functional equation in several variables on Banach modules," Nonlinear Analysis: Theory, Methods \& Applications, vol. 72, no. 3-4, pp. 1755-1767, 2010.

[10] J. Sikorska, "Exponential functional equation on spheres," Applied Mathematics Letters, vol. 23, no. 2, pp. 156-160, 2010.

[11] J. Aczél and J. Dhombres, Functional Equations in Several Variables, Cambridge University Press, Cambridge, UK, 1989.

[12] S. Czerwik, Functional Equations and Inequalities in Several Variables, World Scientific Publishing, River Edge, NJ, USA, 2002.

[13] D. H. Hyers, G. Isac, and Th. M. Rassias, Stability of Functional Equations in Several Variables, Birkhäauser, Boston, Mass, USA, 1998.

[14] Pl. Kannappan, Functional Equations and Inequalities with Applications, Springer, 2009.

[15] P. W. Cholewa, "Remarks on the stability of functional equations," Aequationes Mathematicae, vol. 27, no. 1-2, pp. 76-86, 1984.
[16] S. Czerwik, "On the stability of the quadratic mapping in normed spaces," Abhandlungen aus dem Mathematischen Seminar der Universität Hamburg, vol. 62, no. 1, pp. 59-64, 1992.

[17] S.-M. Jung and P. K. Sahoo, "Hyers-Ulam stability of the quadratic equation of Pexider type," Journal of the Korean Mathematical Society, vol. 38, no. 3, pp. 645-656, 2001.

[18] J. R. Lee, S.-Y. Jang, C. Park, and D. Y. Shin, "Fuzzy stability of quadratic functional equations," Advances in Difference Equations, vol. 2010, Article ID 412160, 16 pages, 2010.

[19] M. S. Moslehian, K. Nikodem, and D. Popa, "Asymptotic aspect of the quadratic functional equation in multi-normed spaces," Journal of Mathematical Analysis and Applications, vol. 355, no. 2, pp. 717-724, 2009.

[20] F. Skof, "Local properties and approximation of operators," Rendiconti del Seminario Matematico e Fisico di Milano, vol. 53, no. 1, pp. 113-129, 1983.

[21] W.-G. Park and J.-H. Bae, "On a bi-quadratic functional equation and its stability," Nonlinear Analysis: Theory, Methods \& Applications, vol. 62, no. 4, pp. 643-654, 2005.

[22] K. Ciepliński, "On the generalized Hyers-Ulam stability of multi-quadratic mappings," Computers \& Mathematics with Applications, vol. 62, no. 9, pp. 3418-3426, 2011.

[23] K. Ciepliński, "On multi-Jensen functions and Jensen difference," Bulletin of the Korean Mathematical Society, vol. 45, no. 4, pp. 729-737, 2008.

[24] K. Ciepliński, "Stability of the multi-Jensen equation," Journal of Mathematical Analysis and Applications, vol. 363, no. 1, pp. 249-254, 2010.

[25] K. Ciepliński, "Generalized stability of multi-additive mappings," Applied Mathematics Letters, vol. 23, no. 10, pp. 12911294, 2010.

[26] K. Ciepliński, "Stability of multi-additive mappings in nonArchimedean normed spaces," Journal of Mathematical Analysis and Applications, vol. 373, no. 2, pp. 376-383, 2011.

[27] W. Prager and J. Schwaiger, "Multi-affine and multi-Jensen functions and their connection with generalized polynomials," Aequationes Mathematicae, vol. 69, no. 1-2, pp. 41-57, 2005.

[28] W. Prager and J. Schwaiger, "Stability of the multi-Jensen equation," Bulletin of the Korean Mathematical Society, vol. 45, no. 1, pp. 133-142, 2008.

[29] C.-G. Park, "Multi-quadratic mappings in Banach spaces," Proceedings of the American Mathematical Society, vol. 131, no. 8, pp. 2501-2504, 2003. 


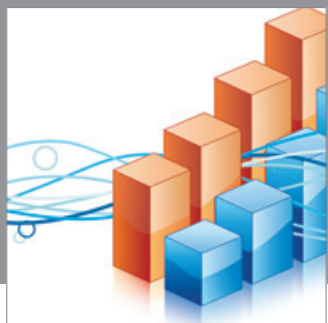

Advances in

Operations Research

mansans

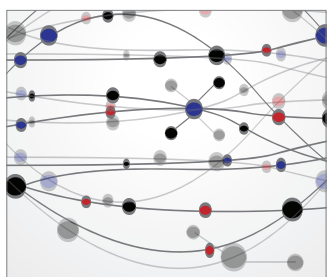

The Scientific World Journal
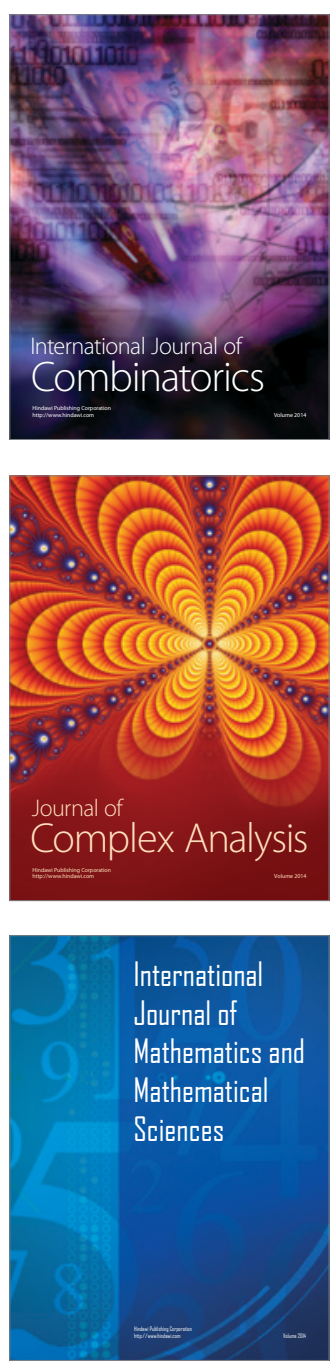
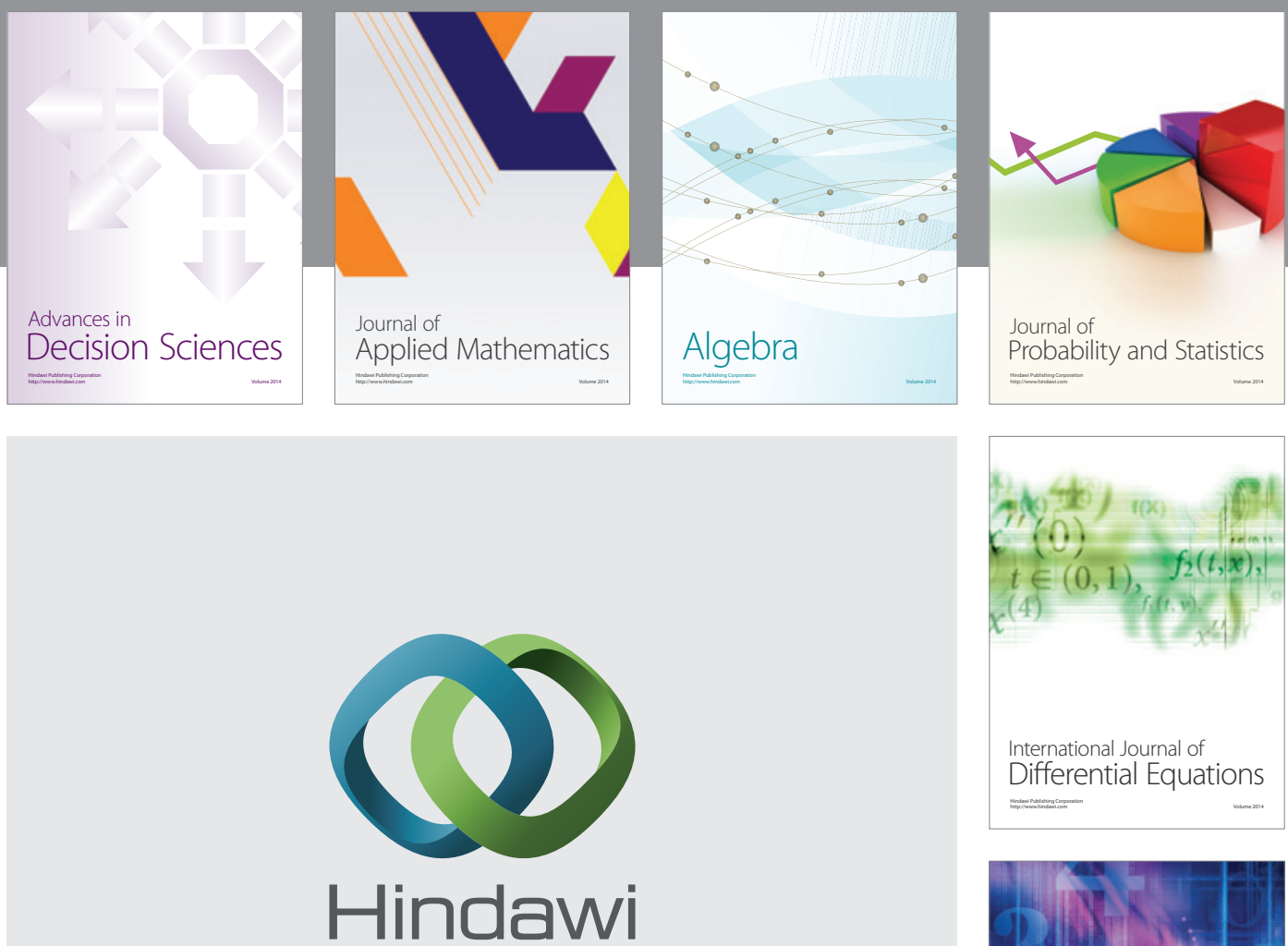

Submit your manuscripts at http://www.hindawi.com


Journal of

Function Spaces

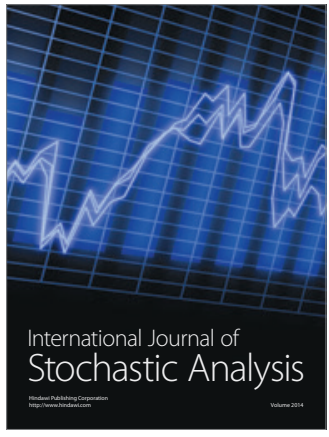

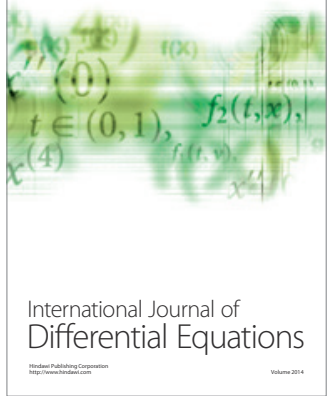
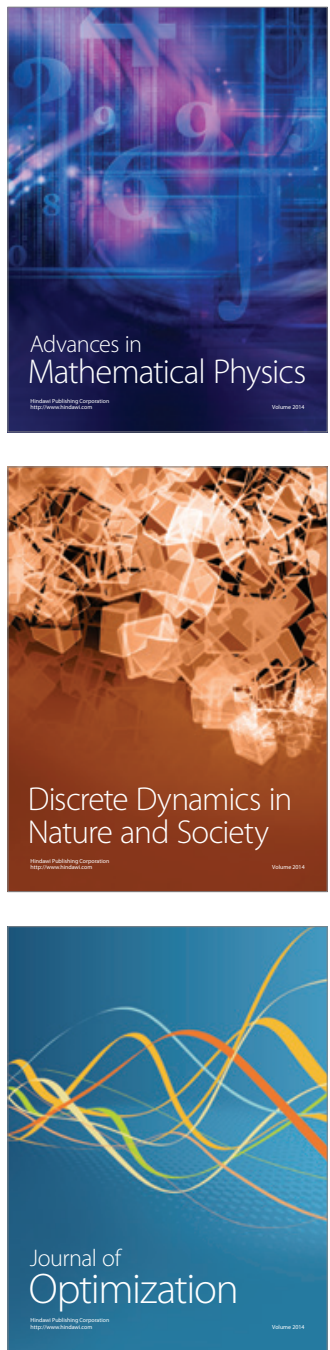